\title{
Erratum to: Simultaneous Positivity for Anti-DNA, Anti-Nucleosome and Anti-Histone Antibodies is a Marker for More Severe Lupus Nephritis
}

\author{
Manshu Sui • Qingyuan Lin - Zhaozhen Xu • Xiaojing Han • \\ Rujuan Xie $\cdot$ Xiuzhi Jia $\cdot$ Xiaofang Guo $\cdot$ Weihua Zhang • \\ Xiuru Guan • Huan Ren
}

Published online: 16 December 2012

(C) Springer Science+Business Media New York 2012

\section{Erratum to: J Clin Immunol}

\section{DOI 10.1007/s10875-012-9825-6}

The original publication of this article contained an error in the first author's name. The name is corrected in the author list above.

\footnotetext{
The online version of the original article can be found at http://dx.doi.org/ 10.1007/s10875-012-9825-6.

M. Sui $\cdot$ X. Han $\cdot$ R. Xie $\cdot$ X. Guo

Department of Nephrology,

First Hospital Affiliated to Harbin Medical University,

150001 Harbin, China

Q. Lin $\cdot$ X. Jia $\cdot$ H. Ren

Department of Immunology, Harbin Medical University,

157 Baojian Road,

Harbin 150081, China

Q. Lin $\cdot$ X. Jia $\cdot$ H. Ren $(\triangle)$

Immunity \& Infection Key laboratory of Heilongjiang Province,

150081 Harbin, China

e-mail: huanren2009@126.com

H. Ren

e-mail: renhuan@ems.hrbmu.edu.cn

Z. Xu $\cdot$ X. Guan

Department of Clinical Laboratory Medicine,

First Hospital Affiliated to Harbin Medical University,

150001 Harbin, China

W. Zhang

Department of Pathophysiology, Harbin Medical University,

150081 Harbin, China 